\title{
Another Landau sum made good
}

The long-standing problem of hydrogen atoms in a magnetic field seems to have been remarkably made plain by a distinctive collaboration between theoreticians and experimentalists.

ONE of the oddest features of the natural world we live in is that some of the simplest problems cannot be simply solved. The three-body problem is the most familiar: there is no closed expression for the motion of three bodies in gravitational interaction with each other except for special cases. And the device of supposing that the mass of one of the three is much smaller than those of its partners is no solution, but merely a means of making the smallest into a test-particle whose trajectory plots the known gravitational field of the other two.

It is almost as surprising, a good sixty years after the late Lev Landau looked at the problem, that there is so little that can be simply said about the behaviour of the simplest atom, that of hydrogen, when immersed in a uniform magnetic field. The question is that of what happens to the motion of the single electron in these circumstances. (Supposing the proton at the nucleus to be infintely massive may simplify the algebra, but does not rid the problem of its complexity.) The surprise is that there is little that is simple that can be said, but the reason is more readily understood.

The simply soluble problems of quantum mechanics are those in which (if necessary, by a suitable transformation of coordinates) Schrödinger's equation becomes a hierarchy of three equations for each particle, each of which relates a momentum to functions of the corresponding (or conjugate, in the hamiltonian sense) space coordinate and to no others. Plainly, the presence of a significant magnetic field must undermine easy expectations of this kind, for the force (or rate of change of momentum) on a moving electron is perpendicular both to the field and to the direction of motion. That is why, as physicists would discover, the equations are not separable or, as mathematicians would prefer to say, the differential equation is not integrable.

It is not surprising that the practitioners should have a sense of being challenged by this state of affairs, but that does not explain why the problem of a hydrogen atom in a magnetic field has become as fashionable as in the past few years. Part of the explanation is that, especially when the electron is mostly a long way from the nucleus (as in an excited or 'Rydberg' state), its behaviour may be described as a delicate mixture of classical and quantum behaviour.
Still more compelling, it has also become clear that even the classical problem (with point-like electrons) behaves chaotically when the electron is only loosely bound. The explanation is that such an electron will move in orbits enormously stretched-out in the direction of the magnetic field, so that small differences of starting-conditions will be enormously magnified as time goes on. Who can resist the study of a system in which the correspondence of chaos in the classical version of a problem is mirrored by the complexity of the quantum version of the problem?

Even so, there is more than that to admire in two articles now in last week's issue of Physical Review Letters (14 January) in which a group from Paris first calculates the properties expected in a magnetic field from hydrogen atoms just below and just above the ionization threshold and then, together with a group from MIT, describes and interprets the results of measurements on the same system.

The theoreticians are D. Delande, A. Bommier and J. C. Gay, from the Ecole Normale Supérieure $(\mathbf{6 6}, 141 ; 1991)$. The experimentalists are Chun-ho Iu, George R. Welch, Michael M. Kash and Daniel Kleppner $(66,145 ; 1991)$. There is no internal evidence to show whether one must admire the theoreticians for their restraint in publishing only when the experiments were complete or the experimentalists for taking on trust the theoreticians' promise that their calculations would be feasible. Either way, the agreement between calculation and experiment is remarkable.

So are the experiments themselves. The MIT group has worked with lithium-7 rather than with hydrogen (which, at least for highly excited or unbound states, should make very little difference to the interactions). The trick is to use a superconducting solenoid to generate a field of 6 tesla $(6,000$ gauss) at which, the calculations show, interesting effects should become apparent. By generating an atomic beam along the axis of the solenoid and with the help of a little laser spectroscopy (with resolution of $10^{-3} \mathrm{~cm}^{-1}$ ), the outcome is essentially an energy spectrum of the system looking for all the world like some spikey noise record. But it is not noise, for the measured spectrum agrees in an uncanny way with the calculated spectrum.
This, broadly, is what one would expect from Landau's work all those years ago. Precise calculations such as those now described were beyond the facilities at his disposal, but the physics of a complicated problem are often most apparent from the crudest approximations. And the most drastic approximation to the behaviour of a hydrogen electron is that in which the atomic nucleus is ignored, reducing the problem to that of the motion of a free electron in a magnetic field.

Unsurprisingly, all possible values of the energy are possible, but Landau's treatment made plain the contribution to the total energy of kinetic energy representing motion along the direction of the field (which is not quantized in this version of the problem) and that at right angles to it (which is quantized in units of $e \hbar H / m c$, where $H$ is the magnetic field and the other quantities refer to the mass and charge of the electron, Planck's constant and the velocity of light in the usual way).

These are still known as the 'Landau levels'. The next approximation to a true atom is then merely to impose the condition that the electron is geometrically confined, when the uncertainty principle can be used to select from among the otherwise multiply infinite array of Landau levels. And, after that, further refinement is possible by taking the electrostatic interaction between the proton and the electron as a perturbation.

Plainly the difficulty for a hydrogen atom near the ionization threshold is that, even in the absence of a magnetic field, there is an infinite number of energy levels, each differing from the previous level by a decreasing amount of energy, but corresponding to a very different and more extended orbit, beyond which there must lie a continuum of accessible states. So how does that system in a free atom mate with the system of energy-levels for a free electron in a magnetic field?

The calculators have set about their task with rigour, even zeal. At one stage they mention, almost in passing, that their approximation to the wave function of an unbound electron consists of an expansion involving 90,000 suitably chosen basis functions. At another, they note laconically that calculating rather less than ten per cent of the spectrum which is their main result occupied a Cray-2 machine for 2,000 seconds - rather more computer power than a PC buff could expect to use up in a lifetime.

John Maddox 